\title{
低损耗 $\mathrm{Ti}$ 扩散 $\mathrm{LiNbO}_{3}$ 光波导
}

\section{李玉善 刘洪举 任秉复 于荣金}

(中国科学院长春物理研究所)

$\mathrm{LiNbO}_{3}$ 是集成光学领域中已经广泛研究的材料. 它可以制作良好的光波导以及优质的 各种元件(除光源和探测器外)。1 1974 年 Schmidt 和 Kaminow ${ }^{[1]}$ 对 Ti 扩散 LiNbO, 光波导进 行了大量的研究,主要是想获得低损耗的单模光波导.

钛内扩散一般是在流动 Ar、 $\mathrm{N}_{2}$ 、空气或真空中进行 ${ }^{[2]}$. 但在高的扩散温度下, 钝内扩散到 $\mathrm{LiNbO}_{3}$ 中去的同时, 整个表面存在着 $\mathrm{Li}_{2} \mathrm{O}$ 的外扩散, 从而使片子表面偏离晶体的化学计量,增 加了非寻常折射率. 结果, 除形成的 $\mathrm{Ti}$ 内扩散波导外, 还存在由 $\mathrm{Li}_{2} \mathrm{O}$ 外扩散形成的平面波 导. 外扩散波导也能束缚在 $y$ 切的 $\mathrm{LiNbO}_{3}$ 片子上沿 $x$ 轴传播的 $T E$ 偏振波，导致信道之间 的干扰. 外扩散引起的晶格空位,还会增加波导的散射损耗. 已有一些人报道了克服 $\mathrm{Li}_{2} \mathrm{O}$ 外 扩散问题的一些方法 ${ }^{[3-7]}$.

\section{一、 $\mathrm{Ti}$ 扩散 $\mathrm{LiNbO}_{3}$ 光波导的制备}

本文用高频贼射的方法, 在仔细抛光的 $y$-切 $\mathrm{LiNbO}_{3}$ 衬底上淀积一层针 (纯度 99.99\%) 膜. 制备的膜厚范围为 $192-920 \AA$, 对大多 数样品的扩散处理为 $1000^{\circ} \mathrm{C}$ 下 8 小时.

为抑制 $\mathrm{Li}_{2} \mathrm{O}$ 的外扩散，我们采用如图 1 所示的闭管扩散系统. 密闭长方形 $(77 \times$ $23 \times 21 \mathrm{~mm}^{3}$ ) 白刚玉舟内放有与样品衬底 相同化学配比的 $\mathrm{LiNbO}_{3}$ 粉和践射过 $\mathrm{Ti}$ 的 $\mathrm{LiNbO}_{3}$ 片子，每次放 $\mathrm{LiNbO}_{3}$ 粉的量为 $0.5-$ 1 克.

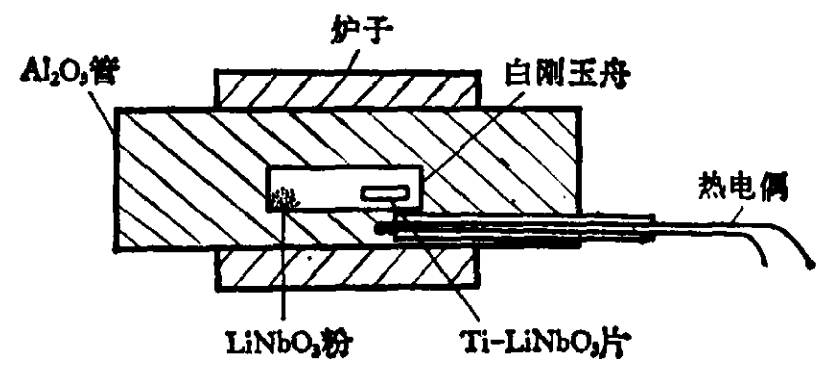

图 1 用于 $\mathrm{Ti}$ 内扩散的装置

表 1 制作波导的条件

\begin{tabular}{|c|c|c|c|c|c|}
\hline 样 品 & $\mathrm{Ti}$ 膜厚度 $(\boldsymbol{A})$ & 扩散温度 ( & 扩散时间(小时) & $\mathrm{LiNbO}_{3}$ 粉 & 导模数 (6328 Å) \\
\hline 2 & 192 & 1000 & 8 & 没有计量 & 单 (TE、 $\mathrm{TM}$ ) \\
\hline 6 & 256 & 1000 & 8 & 新放 0.5 克 & $\begin{array}{l}\text { 单 (TE、TM) } \\
\text { 双 }(\mathrm{TE}, \mathrm{TM}) 4416 \AA\end{array}$ \\
\hline 8 & 690 & 1000 & 8 & 新放 0.5 克 & 双 $(\mathrm{TE}, T \mathrm{M})$ \\
\hline 11 & 920 & 1000 & 13 & 新放 1 克 & 参 $(\mathrm{TE} 、 \mathrm{TM})$ * 未扩尽 \\
\hline 12 & 460 & 1000 & 8 & 新放 0.5 克 & 单 $(T E 、 T M)$ \\
\hline 14 & 690 & 1100 & 8 & 新放 0.5 克 & 双 (TE, TM) \\
\hline
\end{tabular}

本文 1979 年 11 月9 日收到。 
为了检查有无 $\mathrm{Li}_{2} \mathrm{O}$ 外扩散, 制备了 12 号样品. 样品的一半有. $\mathrm{Ti}$ 扩散膜, 而另一半没有 $\mathrm{T}_{\mathrm{i}}$ 扩散膜. 用两个金红石棱镜作光种合输入一输出实验 ${ }^{[3]}$. 结果证明样品表面 $\mathrm{Li}_{2} \mathrm{O}$ 外扩散确 实得到了抑制. 在温度为 $1000^{\circ} \mathrm{C}$ 、时间为 8 小时的扩散条件下发现：Ti 膜厚度在 192-460 范围内, 可获得单模波导 $(6328 \AA)$.

\section{二、光波导提耗测至}

损耗测量是把输出棱镜放在离输入棱镜(输入棱镜固定不变)不同的距离上做的. 用光电 倍增管接收输出讯号, 并作为输出棱镜位置的函数加以记录. 光源为 He- Ne 激光器 (6328 $\AA$ ). 输出棱镜的空气间陌每次尽量一致,并对几个样品反复测量. 光波导的损耗为 $1 d B / \mathrm{cm}$ 左右. 这些结果与观察到的宏观现象一致. 片子从外表上看,是透明的. 用金红石棱镜把 $\mathrm{He}-\mathrm{Ne}$ 激 光耦合进波导时,波导产生的散射极少,并且双模波导的输出 $m$ 线都是单个出现的. 就波导的 制作过程来说，高温扩散以及缓慢降温过程，相当于退火处理。而且，退火又是在放有 $\mathrm{LiNbO}_{3}$ 粉的密闭小舟中进行的，片子表面没有被舟外杂质所沾污。 由表面积比 $\mathrm{LiNbO}_{3}$ 片更大的 $\mathrm{LiNbO}_{3}$ 粉产生的 $\mathrm{Li}_{2} \mathrm{O}$ 蒸气压, 足以与 $\mathrm{Ti}_{1}-\mathrm{LiNbO}_{3}$ 片子保持平衡, 扩散过程有利于减少 $\mathrm{Li}$ 空 位. 上述这些因䒺会使晶体完整性明显增加，从而减少光的散射. 波导样品表面的扫描电镜 照片也证实了这一点. 样品表面很光滑,表面没有位错线。

\section{三、光波导其它头数的测是}

光在扩散波导中的传播,可用下列模色散方程表示:

$$
\Sigma \phi_{i}+2 \phi_{1}+2 \phi_{2}=2 m \pi,
$$

对余误差函数、高斯和指数分布的所有扩散波导,上面的方程可近似地表示成 ${ }^{[8]}$

$$
V \int_{0}^{x_{t}}\left(f(x)-b_{m}\right)^{1 / 2} d x=\left(m+\frac{3}{4}\right) x,
$$

其中, $m$ 是模式数 $(m=0,1,2, \cdots, M)$.

$$
\begin{gathered}
f(x)= \begin{cases}\exp \left(-x^{2}\right) & \text { (高斯函数), } \\
\operatorname{erfc}(x) & \text { (余误差函数), } \\
\exp (-x) & \text { (指数函数), }\end{cases} \\
f\left(x_{t}\right)=b_{m}, \quad x=\frac{y}{d}, \\
V=k_{0} d\left(n_{s}^{2}-n_{b}^{2}\right)^{1 / 2} \quad \text { (归一化扩散深度), } \\
b_{m}=\frac{N_{m}^{2}-n_{b}^{2}}{n_{s}^{2}-n_{b}^{2}} \quad \text { (归一化㮛折射率). }
\end{gathered}
$$

$n_{a}, n_{b}$ 和 $N_{m}$ 分别是波导表面折射率、祄底折射率和模折射率. $k_{0}$ 是自由空间波数 $\left(k_{0}=\frac{2 \pi}{\lambda}\right)$.

$$
d=2 \sqrt{D t} .
$$

对于 $\mathrm{Ti}$ 扩散 $\mathrm{LiNbO}_{3}$ 波导,因为䇅射在 $\mathrm{LiNbO}_{3}$ 表面的量是有限的（一般厚度为几百埃）， 特别当扩散的时间对 $\mathrm{T}_{\mathbf{i}}$ 离子完全进入晶体来说是足足有余的时候, 浓度分布应接近高斯函 数, 这已由电子微探针测量所证实 ${ }^{19}$. 对于低阶模, 可用抛物线分布来近似描述高斯分布, 即:

$$
f(x)=e^{-x^{2}}=1+\left(-x^{2}\right)+\frac{\left(-x^{2}\right)^{2}}{2 !}+\cdots \cong 1-x^{2} .
$$


因此方程 (2) 变成

$$
\begin{gathered}
V \int_{0}^{x_{t}}\left[1-x^{2}-b_{m}\right]^{1 / 2} d x=\frac{V \pi\left(1-b_{m}\right)}{4}=\left(m+\frac{3}{4}\right) \pi, b_{m}=1-\frac{(4 m+3)}{V}, \\
N_{m}^{2}=n_{s}^{2}-(4 m+3)\left(n_{s}^{2}-n_{b}^{2}\right)^{1 / 2} / k_{0} d=n_{s}^{2}-\frac{(4 m+3) \sqrt{ } n_{s}^{2}-n_{b}^{2} \cdot \lambda}{2 \pi d} .
\end{gathered}
$$

$N_{m}$ 可以利用下列方程求出

$$
N_{m}=\cos \theta \cdot \sin \theta_{m}+\sin \theta\left(n_{p}^{2}-\sin ^{2} \theta_{m}\right)^{1 / 2},
$$

其中, $\theta$ 是棱镜角, $n_{p}$ 是棱镜折射率, $\theta_{m}$ 是第 $m$ 阶模式的同步人射角. 实验上测出 $\theta_{m}$ 角, 就 能算出模折射率 $N_{m}$. 表 2 列出了我们实验中算出的数值. 为了比较,在括号中列出了采用双 曲线正割分布 ${ }^{[100}$ (未微扰的)求得的波导参数. 抛物线分布和双曲线正割分布都是对高斯分布 的一种近似. 二种近似的 $n_{s} 、 \Delta n_{s}$ 值差别极小, 而 $d$ 和 $D$ 值差别较大，可能是抛物线分布偏离

\begin{tabular}{|c|c|c|c|c|c|c|c|c|}
\hline \multirow{2}{*}{ 样品 } & \multicolumn{4}{|c|}{$N_{m}$} & \multirow{2}{*}{$n_{s}$} & \multirow{2}{*}{$\Delta n_{s}$} & \multirow{2}{*}{$d(\mu \mathrm{m})$} & \multirow{2}{*}{$D\left(\mathrm{~cm}^{2} /\right.$ 小时 $)$} \\
\hline & $\mathrm{TE}_{\mathrm{o}}$ & $\mathrm{TE}_{\mathrm{i}}$ & $\mathbf{T M}_{\mathbf{0}}$ & $\mathrm{TM}_{\mathbf{2}}$ & & & & \\
\hline \multirow{2}{*}{8} & 2.225 & 2.216 & & & $\begin{array}{c}2.231 \\
(2.232)\end{array}$ & $\begin{array}{c}0.031 \\
(0.032)\end{array}$ & $\begin{array}{c}3.9 \\
(3.3)\end{array}$ & $\begin{array}{c}4.8 \times 10^{-9} \\
\left(3.3 \times 10^{-9}\right)\end{array}$ \\
\hline & & & 2.296 & 2.290 & $\begin{array}{c}2.300 \\
(2.301)\end{array}$ & $\begin{array}{c}0.014 \\
(0.015)\end{array}$ & $\begin{array}{c}4.1 \\
(3.0)\end{array}$ & $\begin{array}{c}5.2 \times 10^{-9} \\
\left(2.8 \times 10^{-9}\right)\end{array}$ \\
\hline \multirow{2}{*}{14} & 2.218 & 2.213 & & & $\begin{array}{c}2.221 \\
(2.222)\end{array}$ & $\begin{array}{c}0.021 \\
(0.022)\end{array}$ & $\begin{array}{c}6.3 \\
(5.6)\end{array}$ & $\begin{array}{c}1.2 \times 10^{-8} \\
\left(9.9 \times 10^{-7}\right)\end{array}$ \\
\hline & & & 2.293 & 2.290 & $\begin{array}{c}2.295 \\
(2.296)\end{array}$ & $\begin{array}{c}0.009 \\
(0.010)\end{array}$ & $\begin{array}{c}5.9 \\
(4.8)\end{array}$ & $\begin{array}{c}1.1 \times 10^{-8} \\
\left(7.2 \times 10^{-9}\right)\end{array}$ \\
\hline
\end{tabular}
高斯分布较大的缘故.

表 2 光波导参数

\section{四、结论}

我们得到了低损耗 $\mathrm{Ti}$ 扩散 $\mathrm{LiNbO}_{3}$ 光波导. 发现: 采用我们实验中闭管扩散的工艺条件 时,波导的制作十分简单，重复性好，既可抑制 $\mathrm{Li}_{2} \mathrm{O}$ 外扩散，又可获得低损耗波导. 因此这种 工艺是合理的. 这对研究和批量生产 $\mathrm{T}_{i}$ 扩散 $\mathrm{LiNbO}_{3}$ 光波导的各种器件也有实际意义.

致谢：刘跃宗同志做了样品的扫描电镜测量,杜淑琴同志帮助淀积金膜,在此表示感谢。

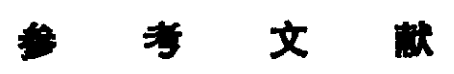

[1] Schmidt, R. V. \& Kaminow, I. P., Appl. Phys. Lett., 25 (1974), 458.

[2] Pan'kin, V. G., Petukhova, S. N., Pchelkin, V. Yu., Shashkin, V. V. \& Shvarts, N. L., Sov. J. Quantum Electron., 8 (1978), 177.

[3] Ranganath, T. R. \& Wang, S., Appl. Phys. Lett., 30 (1977), 376.

[4] Chen, B.-U. \& Pastor, A. C., Appl. Phys. Lett., 30. (1977), 570.

[5] Miyazawa, S., Guglielmi, R. \& Carenco, A., Appl. Phys. Lett., 31 (1977), 742.

[6] Esdaile, R. J., Appl. Phys. Lett., 33 (1978), 733.

[7] Noda, J., Fukuma, M. \& Saito, S., J. Appl. Phys., 49 (1978), 3150.

[8] Hocker, G. B. \& Burns, W. K., IEEE J. Quantum Electron., QE-11 (1975), 270.

[9] Sugii, K., Fukuma, M. \& Iwasaki, H., J. Mater. Sci., 13 (1978), 523.

[10] Kumar, A. \& Khular, E., Optics Communications, 27 (1978), 349. 